\title{
LA EDUCACIÓN (SEXUAL) EN DISPUTA: UN ANÁLISIS DEL CAMPO EDUCATIVO COMO ESCENARIO DE LUCHAS POR LOS DERECHOS DE GÉNERO EN EL URUGUAY DE HOY
}

\section{The (sexual) education in dispute: an analysis of the educational field as a stage of profits for gender rights in Uruguay today}

\begin{abstract}
Nilia Viscardi ${ }^{1}$ Instituto de Educación, Facultad de Humanidades y Ciencias de la Educación, Universidad de la República, Uruguay nilia.viscardi@gmail.com https://orcid.org/0000-0001-8070-3491
\end{abstract}

Leonel Rivero² Facultad de Ciencias Sociales, Universidad de la República, Uruguay

\footnotetext{
1 Responsable científica del proyecto del cual surge la publicación y que fue financiado por la Comisión Sectorial de Investigación Científica de la UDELAR para el período 2021-2023 en el Programa de Investigación y Desarrollo y que radica en el Instituto de Educación. Facultad de Humanidades y Ciencias de La Educación. Universidad de la República. Es actualmente la Directora del Departamento de Pedagogía, Política y Sociedad del Instituto de Educación y la Coordinadora del Doctorado en Educación, ambos en la Facultad de Humanidades y Ciencias de la Educación/UDELAR. Desde el año 2012 desempeña su cargo profesor Agregado, Grado 4, efectivo en la Facultad de Humanidades y Ciencias de la Educación, Instituto de Educación. Departamento de Pedagogía, Política y Sociedad, con un régimen de Dedicación Total radicado en la Facultad de Humanidades y Ciencias de la Educación. Asimismo, desde el año 2010 es Profesora Adjunta Grado 3, efectivo en la Facultad de Ciencias Sociales, Departamento de Sociología, Área de Sociología Política, cuyo cargo se desempeña en Régimen de Dedicación Total radicado en la Facultad de Humanidades y Ciencias de la Educación. A partir del año 2010 es Investigadora Nivel I del Sistema Nacional de Investigadores, ANII.

2 Candidato a Doctor en Sociología por la Universidad de la República (UdelaR), Master y Diplomado en Educación por la Universidad ORT Uruguay, Licenciado en Sociología y Diplomado en Juventud y Políticas Públicas por la UdelaR. Docente e investigador en el Departamento de Sociología (DS-FCS-UdelaR) en el área de trabajo docente y convivencia escolar.
} 
Clarisa Flous ${ }^{3}$

Instituto de Educación, Facultad de Humanidades y Ciencias de la Educación, Universidad de la República,

Uruguay

cfllous@gmail.com

http://orcid.org/0000-0003-0795-3915

Malena Zunino

Instituto de Educación, Facultad de Humanidades y Ciencias de la Educación, Universidad de la República,

Uruguay

malenazunino@gmail.com

ttps://orcid.org/0000-0001-5354-6144

Verónica Habiaga 5

Instituto de Educación, Facultad de Humanidades y Ciencias de la Educación, Universidad de la República,

Uruguay

vhobiaga@gmail.com

https://orcid.org/0000-0002-9727-124X

Recibido:21 de septiembre de 2020

Aceptado: 30 de abril de 2021

\section{Resumen}

Este artículo propone pensar el lugar de la Educación Sexual en el sistema educativo uruguayo; desde el impulso traducido en la política educativa implementada en el "ciclo progresista" (2005-2019) (Noceto,

3 Docente Investigadora del Instituto de Educación. Facultad de Humanidades y Ciencias de la Educación (Udelar).

4 Cargo centrado en la investigación en el Instituto de Educación de la Facultad de Humanidades y Ciencias de la Educación de la Universidad de la República (UdelaR) integrante del grupo: La educación (sexual) en disputa: un análisis del campo educativo como escenario de luchas por los derechos de género en Uruguay. Maestranda en Estudios Sociales Latinoamericanos, Facultad de Ciencias Sociales, Universidad de Buenos Aires (UBA). Licenciada en Sociología, Facultad de Ciencias Sociales, Universidad de la República (UdelaR).

5 Cargo centrado en la investigación en el Instituto de Educación de la Facultad de Humanidades y Ciencias de la Educación de la Universidad de la República (UdelaR) integrante del grupo: La educación (sexual) en disputa: un análisis del campo educativo como escenario de luchas por los derechos de género en Uruguay. Doctoranda en Educación, Facultad de Humanidades y Ciencias de la Educación,UdelaR, Uruguay. Magíster en Estudios Latinoamericanos, Facultad de Humanidades y Ciencias de la Educación en Universidad de la República (UdelaR). 
Piñero y Rosenblat, 2020) y su freno $0^{6}$ impuesto por los recortes y la política del gobierno que inició en 2020. El "impulso y su freno" ratifican a la educación sexual como campo en disputa. Desde las constelaciones discursivas hegemónicas, la educación sexual en la escuela resulta una afrenta a la "libertad" de las familias en relación a la educación de sus hijos. Esta se dice amenazada por el avance de la "ideología de género", construcción atribuida a los distintos movimientos sociales que proponen la revisión del "orden natural" sobre la identidad. Se concluye que, mientras en los períodos anteriores la educación sexual (ES en adelante) aparece asociada a un paradigma médico-sanitario, con el progresismo se constituye en clave de derechos políticos asociados a la identidad y diversidad.

Palabras clave: Educación sexual - currículum escolar - derechos género - diversidad

\section{Abstract}

In this article, we analyze Sex Education in the educational system in Uruguay, as a field in dispute by social actors. To do this, we carry out a historical tour of the main demands of social movements, and the changes in regulations and school curriculum, particularly in the last two decades, marked by the rise and fall of progressivism in the Uruguayan government. It is concluded that, while in previous periods the discussion about sexuality in the educational field appears associated with a medicalhealth paradigm, with progressivism it constitutes the key to political and citizen rights associated with identity and diversity. Likewise, this shift is currently challenged by demands from religious and conservative organizations who seek to exclude sex education from school curriculum.

Keywords: Sex education - school curriculum - rights - gender - diversity

6 Utilizamos el nombre de la obra de Carlos Real de Azúa (1964), considerado como el impulsor de la ciencia política en Uruguay. 


\section{La educación sexual como campo de lucha generacional y de género}

La educación condensa importantes luchas sociales que buscan imprimir el sentido de lo que debe ser educado, aquellos contenidos que forjarán las mentalidades de las próximas generaciones (Durkheim, 1974). El currículum escolar, en particular, ha sido históricamente objeto de luchas entre actores (Benavot, 2006), tradicionalmente desde aquellos que postulan que la educación solo debe impartir "lo básico" (matemáticas y lengua), a quienes amplían el espacio educativo a las artes y otras esferas de lo humano (Eisner, 1972). En esta lucha por la ampliación de la esfera educativa, aparecen nuevas temáticas en discusión. La cuestión sobre el gobierno de los cuerpos y las almas, las pasiones y deseos comienzan a ser demandados como objeto de educabilidad, lo que aparece tematizado en el campo escolar como ES. Así, según Darré:

"la educación sexual (...) no puede definirse a partir de los objetos que convoca. Forma parte del discurso pedagógico, dado que supone una sistematización de ciertos conocimientos en un tiempo determinado. Lleva implícita una selección y distribución de ideas y nociones que serán consideradas legítimas, señalando, en el mismo acto, a ciertos sujetos que se apropiarán del saber y a otros que lo transmitirán desde una posición legítima" (Darré, 2008, p. 202).

La ES en la escuela se constituye como campo en disputa porque en ella se amalgaman supuestos invisibilizados que pugnan por la hegemonía del cuerpo. Pensar en la ES, implica leer los pliegues de la historia de un proyecto escolar que dio sentido. El proyecto pedagógico moderno se estructuró bajo los designios de una «normalidad» que aspiraba a borrar cualquier rastro de «barbarie» significada por el vicio, el ocio y los placeres carnales (Barrán, 1991). Por medio de la "policía de los enunciados" y una economía restrictiva, se delimita una forma de hacer sobre el cuerpo mediante el

"Control, también de las enunciaciones: se ha definido de manera mucho más estricta dónde y cuándo no era posible hablar del sexo; en qué situación, entre qué locutores, y en el interior de cuáles relaciones sociales; así se han establecido regiones, si no de absoluto silencio, al menos de tacto y discreción: entre padres y niños, o educadores y alumnos..." (Foucault, 1998, p. 26).

La escuela y su relación con "lo sexual" ha estado históricamente atravesada por significaciones de "lo prohibido", aquello que precisa invisibilizar o silenciar mediante mecanismos que sentencian la "anormalidad" y su traducción en la culpa (Foucault, 1998, p. 40). En definitiva, la opacidad que habita el abordaje de la "Educación Sexual" muestra que la dificultad no sólo se sitúa en un proceso de borramiento de la "sexualidad" sino en los discursos que se construyen en torno a ella. Esto es, el 
conjunto de significaciones que delimitan un orden natural que ordena la sociedad (Wittig, 1992, p. 33).

Algunas de estas tensiones en torno a la tematización de la sexualidad y su incorporación al sistema educativo se han presentado en el Uruguay. Quisiéramos, a partir de un breve desarrollo histórico, describir dicho proceso, así como algunos actores pujantes, tanto para su incorporación, como exclusión del currículum escolar.

\section{Breve recorrido histórico de la educación sexual en Uruguay}

La institucionalización de la ES en Uruguay constituye un proceso de larga duración cuya génesis se ubica en los albores del siglo XX. Específicamente, se identifican 4 mojones históricos donde los intentos por insertar la ES en el sistema educativo se profundizaron (Darré, 2005; 2008).

El primer momento se ubica alrededor del Novecientos que caracterizado por un contexto nacional optimista y cosmopolita (Caetano, 2010), la sexualidad se constituyó como clave para alcanzar sus impulsos modernizadores. En definitiva, la sexualidad (como elemento fundamental de la sensibilidad) y el modo de producción quedaron entrelazados, alimentando y necesitándose mutuamente (Barrán, 1991). Pensada desde enfoques controladores y disciplinadores (Darré, 2005; 2008) el objetivo de la ES radicó en inculcar el respeto a las normas, la castidad y la abstinencia.

En paralelo a lo que ocurría en la región, las corrientes teóricas en las que se basó su configuración fueron principalmente las cientificistas y positivistas, por lo que el higienismo y la enseñanza biológica-eugenésica fueron movimientos científicospolíticos de gran influencia para la construcción de sentidos sobre la sexualidad y la ES. La ES se introdujo en el parlamento en 1921 con un proyecto de ley presentado por el diputado colorado Mateo Legnani y que el parlamento terminó desaprobando, dejando por fuera del sistema educativo a la ES. Así como el Uruguay se alejaba de ser la "Suiza de América", y veía descensos en su modelo de modernidad, progreso y prosperidad, lo mismo ocurría con la ES: "Si la génesis (creación) del proyecto coincide con ese período, su caída corresponde a otro momento histórico del país y del contexto internacional" (Darré, 2005, p. 93).

El segundo momento se caracteriza fundamentalmente por el Plan Estable creado por Clemente Estable (docente e investigador en biología y neurobiología), por el Programa para Escuelas Rurales en 1949 y el Programa para Escuelas Urbanas en 1957. Con el Plan Estable (1939), instituido de forma experimental entre 1930 y 1970, la sexualidad quedó escindida de los sentimientos y las pulsiones (incluso desde entendimientos negativos de los años anteriores) pasando a estar exclusivamente ligada a una tendencia biologicista y naturalista.

En 1949, se habilitó a las Escuelas Rurales a intervenir en cualquier caso que se detectara como "dificultad sexual"y se incorporó la enseñanza de la reproducción de los seres vivos, vinculada a la higiene y a las conductas desviadas (Darré, 2005). 
El tercer momento es en la década del 90'. Alí se retomó el debate y por primera vez, se recogieron las demandas sociales de la época. Durante la gestión del presidente Luis Alberto Lacalle (1990-1995) nació desde el colectivo docente la demanda de crear un Programa que formase a los y las docentes en ES. Bajo esta propuesta, se alcanzaron a formar 7000 docentes de todo el territorio, llegando a implementarse en modalidad piloto en determinados liceos, cuyo abordaje pretendía ser participativo, diverso, integral y multidisciplinario, tanto en sus supuestos filosóficos y teóricos, así como en la praxis (Benedet y López, 2015). Por consiguiente, esta etapa representa una ruptura en la noción de sexualidad manejada previamente, en tanto desvinculada de analogías al sistema reproductivo de otros seres vivos y desligada a las patologías y desviaciones.

En el marco de tensiones y controversias respecto a la reforma educativa de Germán Rama (Presidente del Consejo Directivo Central del período 1995-2000) y debido al cambio de autoridades fruto del cambio de gobierno, se suspendió el plan en 1995. Según recoge Darré (2005) esto no implicó una defensa activa de Programa, dicha resolución se tradujo en un acatamiento pasivo desde diversos actores sociales, tales como agrupaciones de padres, partidos políticos progresistas ${ }^{7}$, gremios estudiantiles y docentes. Sin embargo, aquel Programa se fundó como el antecedente directo del programa de ES actual, especialmente por su modalidad y lógicas de implementación, donde se destaca que "la educación de la sexualidad puede y debe ser encarada como parte de la práctica y el quehacer docente y pedagógico" (Celiberti, 2005, p.165).

El cuarto momento tuvo lugar durante el gobierno de Jorge Batlle (2000-2005) y se caracterizó por la edición del manual de ES titulado "iEscucha, Aprende, Vive!" (MSP-ANEP-ONUSIDA, 2000) dirigido a estudiantes de tercer año de ciclo básico, elaborada y publicada por el Ministerio de Salud Pública, la Administración Nacional de Educación Pública (ANEP) y ONUSIDA-Programa Nacional de SIDA. A diferencia del Programa anterior, esta guía vuelve a centrarse en la reducción de daños, donde se pondera la prevención de enfermedades venéreas, fundamentalmente el Sida, y los embarazos no planificados.

En función de las complejidades y tensiones, Celiberti (2005) define este período como una "esquizofrenia colectiva". Diversas críticas fueron realizadas desde organismos y organizaciones de la sociedad civil, especialmente por su abordaje en torno a la homosexualidad. La misma fue presentada como una opción sexual aceptada y válida $y$, por ende, exenta de criterios moralizantes "que permitan a los sujetos una identificación normalizada con alguna de ellas" (Darré, 2005, p.153).

La contraofensiva se condensó fundamentalmente en la postura de la Iglesia Católica, que además de manifestar su rechazo hacia los supuestos sobre la homosexualidad

7 Para Sempol (2013), entre 1985 y 2005, los temas de sexualidad no fueron incorporados a las agendas de la izquierda política y social puesto que su discurso hegemónico post-dictatorial no incorporaba la libertad sexual y la crítica a la discriminación como parte de sus demandas. 
-y en menor medida, hacia el autoerotismo-. El cuestionamiento a la laicidad y la falta de neutralidad del texto, se constituyeron como los ataques más potentes. Estas mismas lógicas fueron replicadas en la prensa, tanto en medios escritos como radiales y televisivos, los cuales dieron voz a fundamentos conservadores que precisamente cuestionaban la intromisión de la institución educativa en temáticas cuya potestad la tendrían las familias. En la vereda contraria, se destacaron los apoyos de la Sociedad de Sexología del Uruguay (SUS), La Alianza por la Educación Laica, la Comisión de Educación y Cultura de la Cámara de Diputados e integrantes de la ANEP (Darré, 2005).

Nuevamente las transformaciones estructurales acompañaron las discusiones en torno a la ES. La concepción subyacente a la postura gubernamental privilegiaba a la familia como espacio para hablar de sexualidad, entendida como parte de la esfera privada (Darré, 2005). Bajo este contexto, el manual no llegó a repartirse efectivamente, y su difusión estuvo lejos de la esperada. Una vez más, la ES quedó eliminada del ámbito educativo. Lo mencionado da cuenta de un proceso de larga duración que no es secuencial ni evolutivo, y de enfrentamientos discursivos e ideológicos, donde las disputas sobre la separación entre el espacio público y privado, y la laicidad en la educación aparecen como los puntos neurálgicos de los debates.

En los últimos 15 años, en el marco del ciclo progresista, se asiste a la aprobación de leyes que contribuyen a consolidar derechos sexuales y reproductivos. Estas leyes de alguna forma sintetizan y resultan de una confluencia entre movimientos de jóvenes y de mujeres, y de políticas impulsadas a lo largo de los tres períodos del gobierno progresista (2005 a 2020), aunque no sin tensiones. Dentro de la batería legal de este período se incluye la Ley 18.426 de Defensa del derecho a la Salud Sexual y Reproductiva (2008) ${ }^{8}$ que ubica al Estado como promotor y garante de políticas para el ejercicio de los derechos sexuales y reproductivos (Art l), las cuales, entre múltiples objetivos, tiene la formación de docentes en sus distintos niveles para la educación en el ejercicio de este derecho (Art 2). En 2012, se aprueba la ley sobre Interrupción Voluntaria del Embarazo (IVE) (Ley 18.987) habilitando la despenalización del aborto a partir del cumplimiento de los procesos que la ley plantea.

Las dos últimas leyes en los años 2017 y 2018 cierran el ciclo de "avance de derechos". Por un lado, la ley integral de violencia basada en género y hacia las mujeres (Ley 19.508). Esta ley tiene como objeto garantizar el efectivo goce del derecho de las mujeres a una vida libre de violencia basada en género (Art 1). Por otro, la ley integral para personas trans, aprobada en octubre de 2018 (Ley 19.684) la cual "tiene como objeto asegurar el derecho de las personas trans residentes de la república a una vida libre de discriminación y estigmatización a través de la cuales se establecen

8 Diario de Sesiones Asamblea General Senado y Cámara de Representantes de la República Oriental del Uruguay (2008). Ley 18.426 de Defensa del derecho a la Salud Sexual y Reproductiva, Disponible en https://www.mysu.org.uy/wp-content/uploads/2014/1l/Ley_ No_18-426-DSSRR.pdf 
mecanismos, medidas y políticas integrales de prevención, atención, protección, promoción y reparación" (Art. 1). La ley fue cuestionada a partir de firmas para la de un pre referéndum ${ }^{9}$ a los efectos de ser derogada. La iniciativa no alcanzó los votos, pero explicita elementos conservadores de un cierto sector de la población.

\section{La participación, las movilizaciones por derechos y los estudiantes}

Con relación a la educación, al igual que en Argentina y Chile, las leyes educativas de Uruguay establecen la obligatoriedad del nivel secundario, y también introdujeron una serie de transformaciones normativas en las formas de disciplinamiento escolar, así como en referencia a los derechos sexuales. En el año 2008, se aprueba la Ley General de Educación (Ley n⿳0 18.437) que promueve la participación estudiantil en los centros de enseñanza, a la vez que se impulsaron programas como el de Convivencia saludable. A su vez, el país fue el primero en América del Sur en despenalizar el aborto en el año 2012 y el único que legalizó la producción y consumo del cannabis en el año 2014.

Este proceso de expansión de derechos y ciudadanía ocurrió en el contexto de un movimiento que da origen a dos tipos de dinámicas diferentes frente a la cuestión. Esto es, por un lado, los movimientos que luchan por la consolidación de derechos y, por otra parte, las movilizaciones conservadoras centradas en la recuperación de un discurso moral sobre la familia, el trabajo y la defensa del nacionalismo (Viscardi, 2019). Estas experiencias se corresponden con la vivencia de diferentes formas de construcción de ciudadanía política en la escuela. En algunos casos, subjetividades que anclan en la noción de diálogo y debate en la construcción colectiva de normas escolares, en otros aquellas que construyen jóvenes sin voz, sujetos a la ley en su vertiente penal, con experiencias escolares de acceso desigual a sus derechos y criminalización de sus conductas (Litichever y Núñez, 2015; Morales y López, 2018).

El trabajo de Vaccotti (2019) aporta elementos para pensar la combinación de aspectos novedosos como la resignificación de las prácticas políticas juveniles, así como la emergencia de demandas vinculadas al género. En su estudio sobre el 14 de agosto ${ }^{10}$ como hito y lugar de memoria en el Uruguay, Sempol (2006) destaca que a partir de la década del noventa se plasman diferencias en la construcción de la memoria, así como la conversión de la marcha más en un espectáculo musical (con la inclusión del toque). A su vez, por esos años, varios liceos fueron ocupados en disconformidad con la reforma educativa (Graña, 2005). Luego, en Uruguay, adquiere centralidad el debate en torno a la baja de la edad de imputabilidad penal para

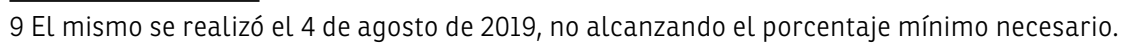


adolescentes de entre 16 y 18 años, a la par de la consolidación de movimientos a favor y en contra con vistas a introducir cambios en la seguridad pública (Viscardi, 2019) que, al igual que con la implementación de la ES en las escuelas, se convierte en un campo de disputa que organiza antagonismos.

A diferencia de lo ocurrido con la Ley de Caducidad, el pedido de baja de edad de responsabilidad penal ancla en una corriente de opinión mayoritaria que se ha estructurado incluso en protestas populares y pedidos de grupos refrendados e impulsados por sectores políticos que aparecen como sus voceros (Mallo, Viscardi y Barbero, 2013) y con la confluencia de movimientos sociales que organizan acciones contra esa criminalización de la juventud. Surgieron exitosos movimientos de reacción al proceso de criminalización juvenil, como la iniciativa "Ser joven no es delito" y el Movimiento "No a la baja" -de la edad de imputabilidad-, ambos espacios aglutinadores de organizaciones y movimientos: juventudes políticas, sindicales, movimientos sociales integrados por jóvenes, entre otros.

\section{La Educación Sexual en la era progresista}

Con la llegada del Frente Amplio al gobierno, la ES se institucionalizó definitivamente en el sistema educativo, abriendo paso a una nueva etapa en la historia nacional, que junto a una batería de leyes y normativas mencionadas anteriormente conformaron la llamada 'agenda de derechos'. Bajo este entendimiento, los Derechos de Salud Sexual y Reproductiva comenzaron a analizarse con perspectiva de los Derechos Humanos y vinculados con la construcción de ciudadanía (Rossi, 2015), haciendo eco de acuerdos internacionales (Carta Universal de los Derechos Humanos; la Convención de los Derechos del Niño y la Convención contra toda forma de discriminación de la mujer) como nacionales (Ley General de Educación, № 18.437, Ley Defensa del Derecho a la Salud Sexual y Reproductiva, No18.426, entre otras).

Durante el "ciclo progresista", que comprende tres periodos de gobierno del partido Frente Amplio, desde el 2005 al 2020, la ES fue entendida como elemento constitutivo del derecho a la educación. Esto se tradujo en una política educativa que habilitó espacios de formación a docentes y estudiantes, al tiempo que se constituyó en una herramienta de producción de conocimiento sobre el tema.

En los gobiernos progresistas se desplegó un conjunto de propuestas desde la ANEP, donde "la educación sexual representa una dimensión de la educación integral de nuestros niños, adolescentes y jóvenes y constituye una estrategia fundamental en la formación de ciudadanos activos y responsables" (ANEP, 2017, p. 9). Asimismo, en el marco del primer gobierno progresista, al amparo de la Ley $N^{\circ} 17.514$ de Violencia Doméstica, se produce uno de los mayores logros del movimiento de mujeres. Esta Ley crea, de acuerdo al Consejo Consultivo Nacional, el Plan Nacional de Lucha contra la Violencia Doméstica.

Ello tuvo impactos en la política educativa, dando origen a tres iniciativas 
fundamentales en materia de Derechos Humanos durante el año 2006, primer año de gestión progresista. En mayo, se crea la Dirección de Derechos Humanos de la ANEP que tiene por cometido llevar adelante los mandatos de esta Ley al interior del Sistema de Educación Pública. Se instalan, asimismo, dos comisiones de relevancia: la "Comisión de Educación Sexual" de la ANEP que originó el Programa de Educación Sexual vigente hasta la fecha y la "Comisión para la promoción de la convivencia saludable, el ejercicio de la ciudadanía y la prevención de la violencia en el marco de una cultura de respeto por los Derechos Humanos". A esto se suma, la derogación del Acta 14, sustituida por un nuevo Estatuto del Estudiante, que mostró una nueva concepción que reconoce el derecho a la participación de los estudiantes en la vida de los centros educativos (ANEP-CODICEN, 2010).

La Comisión de Educación Sexual se conforma por los Consejos de Educación Primaria, Educación Secundaria, Educación Técnico Profesional, por la ex Dirección de Formación Docente, Dirección Central y de la Dirección General de la Salud del Ministerio de Salud Pública (MSP), así como una secretaría técnica encargada de la articulación y organización. Interesa destacar la alianza entre el sector educativo y el sector salud, en tanto "el vínculo entre sexualidad, riesgos y enfermedades ha estado históricamente en los discursos públicos y en las instituciones generando que los programas de ES se instalen en uno u otro organismo con articulaciones entre sí" (Benedet, 2015, p. 170). Allí, se proponía "elaborar un proyecto programático que apunta a la incorporación de la educación sexual en el proceso educativo integral de las y los alumnos, basado en un contexto de Desarrollo, Salud y Derechos que potencie la construcción de ciudadanía". Este incluía una perspectiva teóricoconceptual que articulaba la sexualidad, educación, desarrollo, salud integral y Derechos Humanos (MYSU, 2013).

En la educación media, la ES es obligatoria de primero a cuarto año curricular y se pone a cargo de un docente "referente". La modalidad de trabajo se piensa de forma transversal e integral, con la aspiración de que el Referente sea formador de formadores, y trabaje con docentes en los centros educativos. A cada referente se le destinan 10 horas semanales por institución, y sus contenidos se sujetan a los programas vigentes. Estos elementos implican, en la práctica, la posibilidad de generar una política de ES con amplia cobertura a nivel nacional, influyendo sobre varios aspectos clave para su sustentabilidad: marcos regulatorios, propuesta de implementación y presupuesto.

En el comienzo del tercer, y último, gobierno progresista termina de cristalizarse algunos hitos de este recorrido. Con la aprobación del Plan Nacional de Educación en Derechos Humanos (SNEP, 2016), se instauran diferentes protocolos y mapas de ruta que colocan en el centro la diversidad y multiculturalidad a partir de tres ejes: 1. Incorporación de las variables ascendencia e identidad de género a los sistemas estadísticos, para visibilizar a la población, 2. Otorgar becas, y 3. Capacitar a funcionarios en la temática. 
Pero el elemento de mayor relevancia pública se vincula con la Guía Educativa de Diversidad Sexual elaborada en 2015 por el Ministerio de Desarrollo Social (MIDES) y especialmente a la Propuesta didáctica para el abordaje de la educación sexual en Educación Inicial y Primaria elaborada por el Consejo de Educación Inicial y Primaria (CEIP), junto a la Asociación Civil Gurises Unidos y el Fondo de Población de las Naciones Unidas (UNFPA) en 2017.

\section{Situación de género y sexualidad en la educación hoy}

Los avances permitieron problematizar otros aspectos de la vida escolar, vinculados al género, la diversidad y el bienestar en el aula. A partir de los lineamientos del Plan Nacional de Derechos Humanos, el trabajo en común entre la ANEP y el MIDES da lugar, hacia finales del período progresista, a la primera encuesta de convivencia y discriminación en centros de Educación Media en Uruguay (MIDES-ANEP, 2019). A partir de estos datos, comenzamos a conocer otras aristas del fenómeno, hasta el momento desconocidas. Por ejemplo, que cerca del $16 \%$ del estudiantado no se define heterosexual, solo el $11 \%$ ha conversado sobre el tema con un adulto del centro, y suele conversarlo en mayor medida con amigos fuera del centro educativo que pertenecientes al mismo ( $71,7 \%$ a $50 \%$ respectivamente). Es decir, que aún hoy las cuestiones de género e identidad parecen escapar a la tematización escolar.

Más aún, las cuestiones de género son evidenciadas como soporte de fenómenos de violencia en el espacio educativo (Rivero, 2019). Como se releva en el estudio MIDES-ANEP (2019), el 48,8\% de estudiantes expresan haber sufrido formas de acoso 0 agresión, y 12,5\% señala un componente de tipo sexual, incluyendo comentarios - gestos que les incomodaron, tocar partes del cuerpo sin consentimiento, o realizar algún acto sexual sin consentimiento. Esta situación tiene mayor incidencia en mujeres que en varones ( $51.1 \%$ a $46.2 \%$ ), en estudiantes con situación de discapacidad $(70.5 \%$ frente a $47.3 \%)$ y población LGTBI+ $(57.7 \%$ a $46.7 \%$ de población no LGTBI+). Asimismo, se da cuenta de formas de violencias nulificadoras (silenciamientos), reforzamiento del ejercicio disciplinario sobre adolescentes LGTBI+, falta de información o mensajes negativos sobre diversidad de género, y clasificación y fijación forzada de identidades. Por sobre la prevalencia estadística, quienes vivencian estas formas de acoso y agresión experimentan momentos de gran sufrimiento, miedo e inseguridad, que suponen marcas biográficas.

Por último, de estos estudios (MIDES-ANEP, 2019, Rivero, 2019) se infiere que estas formas de discriminación tendrían efectos en el bajo involucramiento en la institución, un mayor ausentismo, mayor tendencia a la depresión, y menor autoestima. Los centros de estudiantes, en particular, pasarían a ser un lugar privilegiado para la expresión política de demandas, donde las temáticas de 
identidad, orientación sexual y expresión de género pasan a ser temas de agenda. ${ }^{11}$

\section{La ofensiva conservadora}

Uno de los mayores retos en la institucionalización formal de la ES radica en la dificultad para sostenerla en el tiempo (Darré, 2012). Más allá de los problemas dentro del sistema educativo, los avances en la ES en este corto período son fuertemente cuestionados desde fuera, a través de movimientos como "Con mis hijos no te metas"12, Camaleones, Federación de Estudiantes contra la Opresión Ideológica (FECOPRI), Varones Unidos (De Boni et al., 2019), Red de Padres Responsables, así como Iglesias evangélicas, la Iglesia Católica y parte de la bancada de los partidos tradicionales. La potenciación de estos cuestionamientos se desarrolla en el marco de lo que puede ser entendido como "una nueva etapa de la política antigénero en Uruguay" (Abracinskas et al., 2019) que inicia a comienzos del 2017 a partir de la llegada de los autores argentinos Nicolás Márquez y Agustín Laje al país, quienes presentaron su Libro "El libro negro de la nueva izquierda", en el marco del Campamento Beraca, organizado por Misión Vida del pastor Márquez (Abracinskas et al., 2019).

La Guía Educativa de Diversidad Sexual condensó las críticas de aquellos movimientos. Estos arguyen que sus contenidos atentan contra la libertad y el derecho de los padres a decidir sobre la educación de sus hijos. En esa línea, estos cuestionamientos aluden a la violación de la laicidad bajo el entendido que la guía constituye lo que se denomina como Ideología de género (término que surge como reacción a la Conferencia de Naciones Unidas sobre la Mujer en Beijing, 1995).

A diferencia de lo que ocurre con voces análogas de la región, las críticas a la ES en Uruguay no se detienen enfáticamente en las propuestas de promover el aborto, la homosexualidad o la masturbación, sino que estos relatos en Uruguay se basan en la libertad y el derecho de los padres a decidir sobre qué educación brindar a sus hijos e hijas y a los cuestionamientos de la ideología de género (Uval, 2020), cuyo abordaje suele ir de la mano del "adoctrinamiento" o "intento de reclutamiento" por parte de los grupos, organizaciones y personas que impulsan una agenda de Derechos de salud sexual y reproductiva, de igualdad y equidad de género (Abracinskas et al., 2019).

El colectivo "Con mis hijos no te metas" se crea en 2016, en Perú. Se replica en Uruguay donde se define como un movimiento ciudadano que expresa la ideología

11 Franco, Facundo, Por tercer año consecutivo, estudiantes del IAVA reclamaron baños género-neutrales y cambios en normativa de vestimenta, La Diaria Educación, 28 de septiembre de 2017, Disponible en https://Ladiaria.com.uy/educacion/articulo/2017/9/por-tercer-ano-consecutivo-estudiantes-del-iava-reclamaron-banos-genero-neutrales-y-cambios-en-normativa-de-vestimenta/ 
del movimiento que lo sitúa claramente en el marco de las resistencias conservadoras ocasionadas por la expansión de leyes de cuño progresistas en la región. Expresiones similares se encuentran en el colectivo "A mis hijos no los tocan", creado en Uruguay, en 2017, con expresiones nacionales y departamentales, a partir de la crítica explícita a la ya mencionada "Propuesta didáctica para el abordaje de la educación sexual". En agosto de ese mismo año, junto a "Con mis hijos no te metas", organizan una marcha y concentración frente al Ministerio de Educación y Cultura (MEC) con consignas que denunciaban "el adoctrinamiento a la homosexualidad" y proclamas como "a mis hijos los educo yo".

\section{7. ¿Un nuevo ciclo? La alianza neoliberal-conservadora en el gobierno}

Desde el año 2016, se inició el trabajo en el $3^{\circ}$ Congreso Nacional de Educación "Enriqueta Compte y Riqué" que tuvo lugar a lo largo de todo 2017 y cuyos resultados se sistematizan en el Informe final del Congreso Nacional de "Educación Enriqueta Compte y Riqué" (SNEP, 2018). La educación sexual fue un tema ampliamente debatido a nivel nacional y ello se debió principalmente a la reciente difusión de la "Propuesta didáctica para el abordaje de la educación sexual en Educación Inicial y Primaria", elaborada por el Consejo de Educación Inicial y Primaria (CEIP). El material está dirigido al colectivo docente y cuenta con un conjunto herramientas teóricas y prácticas para el abordaje de la Educación sexual en el aula. Asimismo, permite revisar las conceptualizaciones, creencias y supuestos arraigados en los discursos y en las prácticas de los docentes. Propone una secuencia de contenidos programáticos, que tiene como eje el Programa del CEIP (2008) y se desarrolla durante todo el ciclo escolar, desde inicial hasta sexto año. Además, favorece un abordaje integral de la temática desde la articulación de las distintas áreas del conocimiento.

Si bien existieron diversos pedidos e informes que destacan que: "... la educación sexual es una 'herramienta de construcción de ciudadanía' que permite formar personas en la 'integralidad de su condición de ser humano', garantizando que puedan construir su sexualidad en un 'marco de afectividad'" (SNEP, 2018, p.77) también se dieron expresiones que se manifestaron contrarias a que la educación sexual sea una línea transversal de la Ley General de Educación.

En este sentido, existen opiniones contrarias a la perspectiva de género, refiriéndose a la misma como "ideología de género". Estas posturas cuestionan la posibilidad de que se aborde la temática desde la educación pues lo consideran una "violación al derecho de los padres de educar a los hijos en determinadas creencias y valores" (SNEP, 2018). Se plantea que se debe pedir un permiso expreso a las familias para tratar la temática, pues no se debe "problematizar ni censurar en el centro educativo lo que el niño aprende en la casa en materia de sexualidad" (SNEP, 2018, p. 77). En síntesis, aparecía una sociedad dividida y la consulta popular mostró las reticencias existentes por parte de los representantes de las familias para aceptar el impulso 
a los derechos sexuales y reproductivos al interior del sistema de enseñanza. La transformación cultural se mostraba defendida mayormente por los docentes.

Hasta el año 2019, estas iniciativas de tinte conservador se manifestaban como resistencias a un proyecto general de gobierno de avance en derechos de género y diversidad en el espacio educativo. Sin embargo, la elección nacional de ese año marca un cambio en la relación de fuerzas, con el ascenso al gobierno de una coalición de partidos predominantemente neoliberal-conservador. Es así que varias de las organizaciones descritas se encuentran vinculadas a figuras dentro del nuevo partido de gobierno.

¿Cuáles son las nuevas orientaciones concretas en materia educativa? En primer lugar, una marcada orientación privatizadora. Ello no es nuevo, desde los años noventa, las políticas educativas en América Latina son interpeladas por lógicas de privatización. Estas se cristalizan bajo reformas que se introducen mediante la adopción de un modelo de administración y financiación de la educación, ligado a la gestión y algunas formas de evaluación (Andrade, 2009; Martinis, 2020).

En los últimos meses del presente año se impulsó la Ley de Urgente Consideración (LUC), que tiene a la educación como blanco de estos intereses. Mediante una ley que bajo atavíos de "orden" y "austeridad" viene a erosionar al sistema educativo y sus cimientos, se pone en cuestión el valor de lo público y del

Es claro que esa labor traduce la lucha de sectores específicos de la sociedad uruguaya por la construcción de sentidos en el espacio educativo el cual pensamos y proponemos indagar como campo -en el sentido de Bourdieu (1995)-, en el cual diferentes actores buscan legitimar sus intereses y puntos de vista resignificados hoy a la luz de las demandas de género y los derechos sexuales y reproductivos.

Qué sucederá en concreto con la educación sexual no ha sido explicitado. Sin embargo, algunas pistas se trazan, para cuya lectura es necesario realizar algunas mediaciones conceptuales. Es preciso actualizar la investigación en relación a las modalidades que adquiere, en el campo educativo hoy, la lucha en torno al valor de "lo público" (Martinis, 2020). En ese contexto, la LUC marca un antes y un después en la educación uruguaya, ya que impacta directamente los cimientos de la tradición. En el espacio educativo, donde se entreteje el complejo entramado de cohesión social, de ciudadanía y de democracia la escuela ha sido el lugar de construcción de un relato común. Hoy, la disputa sobre ese relato se hace en torno a la noción de laicidad, bajo la cual, como valor, los diferentes actores justifican la defensa de leyes, discursos y acciones en este campo en pugna. La educación sexual, en concreto, es un ámbito práctico, en acción, en que los sentidos e intereses de los diferentes actores se plasma: estudiantes, padres, comunidad educativa, integrantes de asociaciones civiles, partidos políticos, diferentes sectores eclesiásticos, gobierno de la educación, asociaciones docentes, entre otros.

La LUC, impuesta como asunto "urgente", produce un borramiento explícito de Ley General de Educación (LGE), socava el rol del Estado como garante del derecho, 
subvierte la idea de derecho por la de servicio (Romano, 2020) y resignifica la noción de lo público y lo privado. Impulsa otra noción de evaluación, la construcción de proyectos focalizados y nuevas formas de contrato laboral de los docentes. En este sentido, "hay un punto bien claro: la supresión del adjetivo 'público' no es una cuestión retórica, sino que tiene profundas consecuencias" ${ }^{13}$. Así, la educación deja de ser pensada "como un derecho para proponerse como un "servicio", algo que se ofrece en el mercado para quienes puedan adquirirlo (...) trae, además, una fuerte presión sobre el concepto mismo de la educación como derecho humano fundamental" (Aboites, 2009, pp. 71-72).

En segundo lugar, sobre estos cambios normativos de corte privatizador, se suman algunas decisiones ejecutivas de tinte autoritario. Una muestra de ello, refleja la Circular $n^{0}$ 15/ 2020 del CODICEN (Acta $n^{0} 26$, Res, $n^{0} 18$ ), que bajo el supuesto cumplimento de la Laicidad, establecido en el Art. de la Ley General de Educación no 18437; Resuelve: 1- "Asegurar el respeto irrestricto de los principios rectores de la educación pública, en particular el de la laicidad, así como la prohibición de realizar proselitismo de cualquier especie; 2- El retiro de toda cartelería que atente contra Los principios rectores de la educación; 3) que los Consejos de Educación y Formación en Educación desarrollen acciones que permitan concientizar las comunidades educativas de estas temáticas sin perjuicio (...) asegurando el tratamiento integral y crítico de todos los temas..." (Codicen, 2020). Así, con gran vehemencia el Consejo Directivo Central de la ANEP arremetió contra las diversas formas de expresión de movimientos docentes y estudiantiles, particularmente prohibiendo expresiones contra la LUC. De esta forma, en nombre de la "laicidad" se cercenan los espacios de participación y expresión democrática de ideas. Mediante la imposición se procura asegurar la pluralidad y las garantías democráticas.

\section{Conclusiones}

Hoy, en Uruguay y en la región, parece claro que la asunción de sectores conservadores ha abonado la necesidad de eliminar la enseñanza de la ES en la escuela, entendiéndolo como la intromisión del Estado en la vida privada bajo la amenaza de la "ideología de género". Asimismo, pone en tela de juicio cualquier manifestación de colectivos que, con su lucha, habían instalado y resituado la agenda de los derechos que en el marco de los gobiernos progresistas se concretaron en leyes, programas y acciones.

El campo de ES aparece, y apareció en la historia del Uruguay, como un campo en

13 Castro, Leticia, Especialistas advierten sobre "tendencia privatizadora" de la educación en la LUC, 26 de marzo de 2020, Disponible en: https://Ladiaria.com.uy/educacion/articulo/2020/3/especialistas-advierten-sobre-tendencia-privatizadora-de-la-educacion-en-la-luc/ 
disputa con componentes que tocan a una batalla política en torno a la noción de familia y orden sexual. Hemos intentado, en este marco, hacer su recorrido y mapear las tensiones actuales. En estas disputas, lo que está en juego sistemáticamente es el acceso a la ley y a los derechos de género, sexuales y reproductivos. Las características propias del contexto uruguayo han hecho que las demandas por ES partan sobre todo de los docentes, a diferencia del contexto regional en que observamos demandas y acciones impulsadas por el movimiento estudiantil secundario, en particular las relativas a los derechos sexuales y frente a la estigmatización. En Uruguay, el discurso estudiantil se ha centrado en el problema de los jóvenes y de la discriminación.

La disputa, entonces, se juega sobre todo en la relación entre el gobierno de la educación, los docentes y los representantes de los padres y la sociedad civil. Tensionados por las corrientes de opinión, el avance de conjunto logrado en materia de derechos sexuales y reproductivos, determinó una cooptación del discurso conservador atrincherado en la defensa de la laicidad y del derecho a construir la opción sexual de los hijos por parte de los padres. Se trata de un discurso en el cual la construcción de una voz propia en relación a las opciones sexuales y reproductivas de niños, niñas y adolescentes que impulsa el Estado es sentida como una amenaza.

Este giro conservador que se fue consolidando en los últimos tres años tiene ahora la posibilidad de contar con un gobierno que ponga en práctica sus creencias, significando un retroceso en la implementación del Programa de Educación Sexual. Con cambios y permanencias, esta historia muestra la importancia que adquieren los espacios curriculares en los cuales las opciones de niños, niñas y adolescentes sean comprendidas como una elección consciente e informada. Esto es, como un camino en la construcción de su personalidad y sus derechos y no como amenaza a un orden social, sexual y económico que se defiende sobre la base de la restricción de información y la educación a los estudiantes.

Los temas vinculados a los derechos sexuales y reproductivos siguen presentándose como marginales en relación a otros que son entendidos como cuestión de Estado por ser estos percibidos de interés general, tales como la economía, la seguridad pública, la educación, las finanzas, entre otros. Este fenómeno al que Segato (2016) llama "minorización", está asociado a

"la creencia moderna de que todo lo relativo al tema de las relaciones de género es clasificable dentro del ámbito de lo privado y de lo íntimo", en consecuencia "empujado al rincón residual de la gran política, de la gran justicia y de la seguridad" (Segato, 2016, p.103).

Estas lógicas, además de reproducir la infantilización y el desprecio hacia estas demandas, en un sistema adultocéntrico y patriarcal que no quiere dar lugar a sus cuestionamientos y pretende retroceder en los derechos conquistados, ahora se fortalece con relatos que criminalizan a las mujeres y disidencias en un contexto 
regional que experimenta la avanzada conservadora y, junto a ella, la re-emergencia de discursos anti derechos englobados bajo la "ideología de género".

\section{Bibliografía}

Aboites, H. (2009). Derecho a la educación y libre comercio: las múltiples caras de una confrontación. En Gentili, P. y Frigotto, G. (comps.). Políticas de privatización, espacio público y educación en América Latina (pp. 67-82). Buenos Aires, Argentina: CLACSOHomoSapiens.

Abracinskas, L., Puyol, S., Iglesias, N., Kreher, S. (2019). Políticas Antigénero en Latinoamérica. Uruguay, el mal ejemplo. Montevideo, Uruguay: MYSU.

Andrade, D. (2009). Reformas educativas y redefinición de las fronteras entre lo público y lo privado. En Gentili, P. y Frigotto, G. (comps.). Políticas de privatización, espacio público y educación en América Latina (pp. 121-134). Buenos Aires, Argentina, CLACSO: HomoSapiens.

Administración Nacional de Educación Pública (ANEP) (2020). Circular no 5/2020. Recuperado de https://www.utu.edu.uy/sites/www.utu.edu.uy/files/resolucion/ archivos/2020/06/circular-codicen-15-2020.pdf

Administración Nacional de Educación Pública (ANEP) (2007). Breve análisis histórico de la educación en Uruguay. Documento para la discusión. Recuperado de http://www. ceip.edu.uy/documentos/normativa/programaescolar/analisis_historico.pdf

Administración Nacional de Educación Pública (ANEP) (2008). Educación sexual: su incorporación al sistema educativo. Montevideo, Uruguay: Nordan-Comunidad del Sur.

Administración Nacional de Educación Pública (ANEP) (2017). Evaluación del Programa de Educación Sexual. Recuperado de https://uruguay.unfpa.org/sites/default/ files/pub-pdf/Presentaci.pdf

Barrán, J. P. (1991). Historia de la sensibilidad en el Uruguay, tomo 2, El disciplinamiento (1860-1920). Montevideo, Uruguay: Ediciones de la Banda Oriental.

Benavot, A. (2006). La diversificación en la educación secundaria. Currículos escolares desde la perspectiva comparada. Profesorado. Revista de currículum y formación del profesorado, 10 (1), 1-30.

Benedet, L. (2015). La Educación Sexual en el sistema educativo formal durante el período 2005-2009. Análisis desde un enfoque de género y de políticas públicas. En López Gómez, A (coord.). Adolescencia y sexualidad. Investigación, acciones y política pública en Uruguay (2005-2014) (pp. 161-177). Montevideo, Uruguay: Facultad de 
Psicología, Universidad de la República; UNFPA.

Benedet, L. y López, A. (2015). La educación sexual en Uruguay: enfoques en disputa en la genealogía de la política pública. Temas de Educación, 21(1), 11-30.

Bourdieu, P; Pierre, Wacquant, L. (1995). Respuestas. Por una antropología reflexiva. DF, México: Grijalbo.

Caetano, G. (2010). Ciudadanía y nación en el Uruguay del Centenario (1910-1930). La forja de una cultura estatista. Iberoamericana, 10 (39), 161-76.

Cano, A. (2019). Entre el retorno de la CONAE y lo mismo pero gradual. El programa educativo de la coalición multicolor. Hemisferio Izquierdo. Recuperado de https:// www.hemisferioizquierdo.uy/articulos/author/Agust\%C3\%ADn-Cano-ENTREEL-RETORNO-DEL-CONAE

Celiberti, L. (2005). Las sexualidades en los centros educativos. Discursos y prácticas del sistema educativo. En López Gómez, A (coord.). Adolescentes y Sexualidad. Significados, discursos y acciones en Uruguay. Un estudio retrospectivo (1995-2004) (pp. 155-178). Montevideo, Uruguay: Cátedra Libre en Salud Reproductiva, Sexualidad y Género de la Facultad de Psicología-Universidad de la República; UNFPA.

Comisión Organizadora del Debate Educativo (CODE) (2007). Informe Final. Recuperado de http://repositorioddp.mec.gub.uy/jspui/bitstream/repositorio/81/2/CODE\%20 Informe\%20final.pdf

Darré, S. (2005). Políticas de género y discurso pedagógico. Montevideo, Uruguay: Ed.Trilce.

Darré, S. (2008). "Cuando el saber no tiene lugar: la difícil implementación de la educación sexual en el sistema educativo uruguayo" En Araújo, K., Pietro, M. (2008) Estudios sobre sexualidades en América (pp. 199-213). Quito, Ecuador: FLACSO-Ecuador.

Darré, S. (2012). Investigar en educación sexual. No vayas a decir las cosas que te dijimos (Uruguay, 2009 - 2010). Revista Pucara, (24). Recuperado a partir de https:// publicaciones.ucuenca.edu.ec/ojs/index.php/pucara/article/view/132

De Boni, I., Delacoste, G., Naser, L., Outeda, L., Pérez, D., Pérez Castillo, S., y Sánchez, G. (2019). La reacción. Derecha e incorrección política en Uruguay. Montevideo, Uruguay: Estuario Editora.

Durkheim, E. (1974). Educación y sociología. Buenos Aires, Argentina: Schapire.

Eisner E. (1972). Educar la visión artística. Barcelona, España: Paidós.

Foucault, M. (1998). Historia de la sexualidad (1). La voluntad de saber. Buenos Aires, Argentina: Siglo XXI.

Gentili, P. (2011). Pedagogía de la igualdad. Buenos Aires, Siglo veintiuno editores. 
Graña, F. (2005). Nosotros, los del gremio. Montevideo, Uruguay: Editorial NordanComunidad.

Litichever, L. y Núñez, P. (2015). Radiografías de la experiencia escolar. Buenos Aires, Argentina: Grupo Editor Universitario.

López Gómez, A. (coord.) (2005). Adolescentes y Sexualidad. significados, discursos y acciones en Uruguay un estudio retrospectivo (1995 - 2004). Montevideo, Uruguay: Facultad de Psicología, Universidad de la República; UNFPA.

Mallo, S.; Viscardi, N. y Barbero, M. (2013). La protesta social conservadora. Representaciones sociales y nuevas sensibilidades sobre juventud y violencia a través de las movilizaciones en torno a la baja de edad de responsabilidad penal. En Riella, Alberto (Coordinador). El Uruguay desde la sociología XI (pp. 385-402). Montevideo, Uruguay: DS, FCS.

Martinis, P. (2020). Disputas sobre el sentido de la educación pública: el financiamiento de la "educación pública de gestión privada" en Uruguay. Fineduca, 10 (17), 1-19.

Ministerio de Desarrollo Social-Administración Nacional de Educación Pública (MIDES-ANEP) (2019). Convivencia y discriminación en centros de educación media en Uruguay. Recuperado de http://repositorio.mides.gub.uy:8080/ xmlui/bitstream/handle/123456789/1920/Informe\%20convivencia $\% 20 y \% 20$ discriminaci $\%$ c3\%b3n $\% 20$ en $\% 20$ centros $\% 20$ de $\% 20$ educaci $\%$ c $3 \%$ b3n $\% 20$ media\%20en\%20Uruguay.pdf? sequence=1\&isAllowed=y

Ministerio de Salud Pública (MSP), Administración Nacional de Educación Pública (ANEP), ONUSIDA (2000). jEscucha, aprende, vive! Montevideo, Uruguay: Ed. en Taller de Comunicación S.R.L.

Morales, M. y López, V. (2018). Criminalización de la juventud en Chile: políticas de convivencia escolar y traducciones locales. En Tenenbaum, G. y Viscardi, Nilia (Coord.). XX Juventudes y violencias en América Latina. Sobre los dispositivos de coacción en el siglo XXI (pp. 173-184). Montevideo, Uruguay: UCUR.

Mujer y Salud en Uruguay (2013). Educación Sexual. Una construcción permanente. Recuperado de http://www.mysu.org.uy/haceclick/libro-hace-click.pdf

Nocetto, L.; Piñeiro, R. y Rosenblatt, F. (2020). Uruguay 2019: Fin del ciclo progresista y reestructura del sistema de partidos. Revista de ciencia política (Santiago), 40(2), 511538. doi: 10.4067/S0718-090X2020005000117

Diario de Sesiones Asamblea General Senado y Cámara de Representantes de la República Oriental del Uruguay (2008). Ley 18.426 De Defensa de derechos a la Salud Sexual y Reproductiva Publicada D.0. 10 dic/008 - No 2763.

Rivero, L. (2019). Educación técnica profesional en Uruguay. Aproximación al 
análisis de aspectos claves bajo una mirada con enfoque de género [Informe]. Montevideo, Uruguay: CEPAL - INMUJERES - CETP. Recuperado de https:// planeamientoeducativo.utu.edu.uy/sites/planeamientoeducativo.utu.edu.uy/ files/2019-11/Presentaci\%C3\%B3n\%20Consultor\%C3\%ADa\%20ETP\%20-\%20 G\%C3\%A9nero\%20-\%20Uruguay\%20\%281\%29.pdf

Romano, A. (2020). De la reforma educativa al Plan nacional de educación. La Diaria. Recuperado de https://Ladiaria.com.uy/articulo/2020/6/de-La-reformaeducativa-al-plan-nacional-de-educacion/

Sistema Nacional de Educación Pública (2018). Informe final del Congreso Nacional de Educación Enriqueta Compte y Riqué. Recuperado de: http://repositorioddp.mec. gub.uy/jspui/handle/repositiorioddp/169

Rossi, S. (2015). Espacios de Salud Adolescente y derechos sexuales y reproductivos. Un estudio sobre las percepciones de adolescentes y equipos técnicos de servicios públicos. En López Gómez, A (coord.) Adolescencia y sexualidad. Investigación, acciones y política pública en Uruguay (2005-2014) (pp. 139-157). Montevideo, Uruguay: Facultad de Psicología, Universidad de la República; UNFPA.

Segato, R. (2016). La guerra contra las mujeres. Madrid, España: Traficante de sueños.

Sempol, D. (2006). De Líber Arce a liberarse. El movimiento estudiantil uruguayo y las conmemoraciones del 14 de agosto (1968-2001). Jelin, E. y Sempol, D. (Comp.) El pasado en el futuro: los movimientos juveniles (pp. 65 - 103) Buenos Aires, Argentina: Siglo XXI.

Sempol, D. (2013). De los baños a las calles: historia del movimiento lésbico, gay, trans uruguayo (1984-2013). Montevideo, Uruguay: Debate.

Uval, N. Resistencias a la educación sexual en Uruguay. La Diaria, 31 de enero de 2020. Recuperado de https://Ladiaria.com.uy/feminismos/articulo/2020/1/ resistencias-a-la-educacion-sexual-en-uruguay/

Vaccotti, R. (2019). La relación familia-institución educativa en enseñanza media: perspectivas de docentes de secundaria. Pág.de Educ, 12 (1), 164-178. doi 10.22235/ pe.v12il.1787

Viscardi, N. (2019). Algunas razones para el temor: acerca de la importancia de una política de seguridad humana. Hemisferio Izquierdo, 16 de octubre de 2019. Recuperado de https://www.hemisferioizquierdo.uy/single-post/2019/10/16/algunasrazones-para-el-temor-acerca-de-La-importancia-de-una-pol\%C3\%ADtica-deseguridad-human

Wittig, M. (1992). El pensamiento heterosexual y otros ensayos. Barcelona, España: Editorial Egales. 